\title{
NuSTAR observation of the Arches cluster: X-ray spectrum extraction from a 2D image
}

\author{
Roman Krivonos*t \\ Space Research Institute (IKI), Moscow, Russia \\ E-mail: krivonoseiki.rssi.ru
}

The NUSTAR mission performed a long (200 ks) observation of the Arches stellar cluster in 2015. The emission from the cluster represents a mixture of bright thermal ( $k T \sim 2 \mathrm{keV}) \mathrm{X}$-rays and the extended non-thermal radiation of the molecular cloud around the cluster. In this work we describe the method used to decouple spatially confused emission of the stellar cluster and the molecular cloud in the NUSTAR data.

11th INTEGRAL Conference Gamma-Ray Astrophysics in Multi-Wavelength Perspective 10-14 October 2016

Amsterdam, The Netherlands

* Speaker.

${ }^{\dagger}$ A footnote may follow. 


\section{Introduction}

The Arches cluster is a young, densely packed massive star cluster in our Galaxy that shows a high level of star formation activity. It is located in the inner Galactic Center (GC) region at the projected distance of $11^{\prime}$ from the position of the dynamic center of the Galaxy - Sagittarius A*.

The Arches cluster is a known source of thermal and non-thermal X-ray emission. The thermal emission is thought to originate from multiple collisions between strong winds of massive stars $[1,2,3,4]$, and is localized within the core of the cluster that is about $9^{\prime \prime}(\sim 0.35 \mathrm{pc}$ at $8 \mathrm{kpc})$ in radius [5]. Diffuse non-thermal X-ray emission, revealed by its bright fluorescent $\mathrm{Fe} \mathrm{K} \alpha 6.4 \mathrm{keV}$ line emission, has been detected from a broad region around the cluster $[3,6,7,8,9]$.

The core of the Arches cluster has been relatively well studied in the standard $2-10 \mathrm{keV}$ energy band with the Chandra and XMM-Newton observatories. The origin of the non-thermal extended emission, whether it is produced by the photoionization of the cloud by X-ray photons or through excitation by CR particles, was considered in many relevant studies [7, 8, 9]. [10] analysed the long-term behavior of the Arches cloud over 13 years and reported a 30\% decrease in Fe $\mathrm{K} \alpha$ line and continuum flux of the cloud emission in 2012-2013, providing the evidence that the majority of the variable non-thermal emission is due to X-ray reflection. Despite LECR-only emission is almost excluded based on variability, one could expect that steady background level is a result of CR heating, while most of the varying emission is due to reflection. For this reason we continue to measure spectral shape of the Arches cloud non-thermal emission, which contains imprints of the emission mechanism.

To separate thermal emission of the Arches cluster and non-thermal emission of the surrounding molecular cloud with NUSTAR [11], we utilize 2D image analysis as demonstrated in [12]. Similar procedure was also applied by [13], who analysed NUSTAR data of local Seyfert 2 active galactic nucleus (AGN) NGC 5643 and successfully decoupled partially confused spectra of AGN core and ultra-luminous source located in the same galaxy. In this paper we present 2D image spectral extraction procedure in more details.

\section{The standard approach}

The canonical method of spectrum extraction from X-ray data implies selecting circular region around the source and the region with representative source-free background. Then the standard tools of a given X-ray mission extract events from the regions; calculate the exposure; apply corrections for the Point Spread Function (PSF), vignetting, dead-time, etc.; generate appropriate Response Matrix Files (RMFs) and Ancillary Response Files (ARFs); and finally assemble source and background Pulse Height Amplitude (PHA) files with updated header keywords for spectral modeling in one of the popular packages, e.g. XSPEC [14] or SHERPA [15]. The standard approach fails in complicated cases with non-uniform background or spatial confusion of the sources in the X-ray images. The spatial modelling both the emission of the sources and background provides natural solution to this problem, however requires more sophisticated algorithms.

\section{3. $2 \mathrm{D}$ image fitting procedure}

We first combined the NUSTAR data into sky mosaics in 15 energy bands logarithmically cov- 
ering the NUSTAR working energy range $3-79 \mathrm{keV}$. Each data set contains counts and exposure map. In the following analysis we assume flat background, so we do not need non-uniform background map, normally produced as a separate map in the same pixel resolution. Additional map contains PSF extracted from the NUSTAR Calibration Data Base (CALDB), placed in the center of the map. We assume the same PSF shape over the considered energy range $3-79 \mathrm{keV}$.

We then constructed spatial model of the Arches cluster complex, which includes two 2D Gaussians. The first represents the cluster's core emission, with the position fixed at the centroid coordinates measured in [9] and width fixed at 4" FWHM (PSF smearing effect). The second Gaussian was aligned with the corresponding "halo" Gaussian component used in [9] to describe the extended cloud emission, setting the FWHM model parameter at $72^{\prime \prime} .4$. We fixed sky positions of the Gaussians as described in [12]. Thus, the only amplitudes of the 2D Gaussians were free parameters. As mentioned above, we assume flat background over the detector image. The normalization parameter was estimated in the annulus $70^{\prime \prime}<R<130^{\prime \prime}$ around the cluster.

We use SHERPA modeling and fitting package, a part of CIAO software [16], to construct 2D models and fit them to data. SHERPA enables one to construct a complex models from simple definitions, e.g. our current model setup can be expressed as $p s f($ gauss $2 d . G 1+$ gauss $2 d . G 2) *$ emap + const $2 d . b k g *$ emap, where $p s f($ gauss $2 d . G 1+$ gauss $2 d . G 2) *$ emap means convolution of two Gaussians $G 1$ and $G 2$ with the PSF multiplied by the exposure map emap, the constant background term $b \mathrm{~kg}$ is added as const $2 \mathrm{~d} . b \mathrm{~kg} *$ emap. Note that the background is not convolved with the PSF.

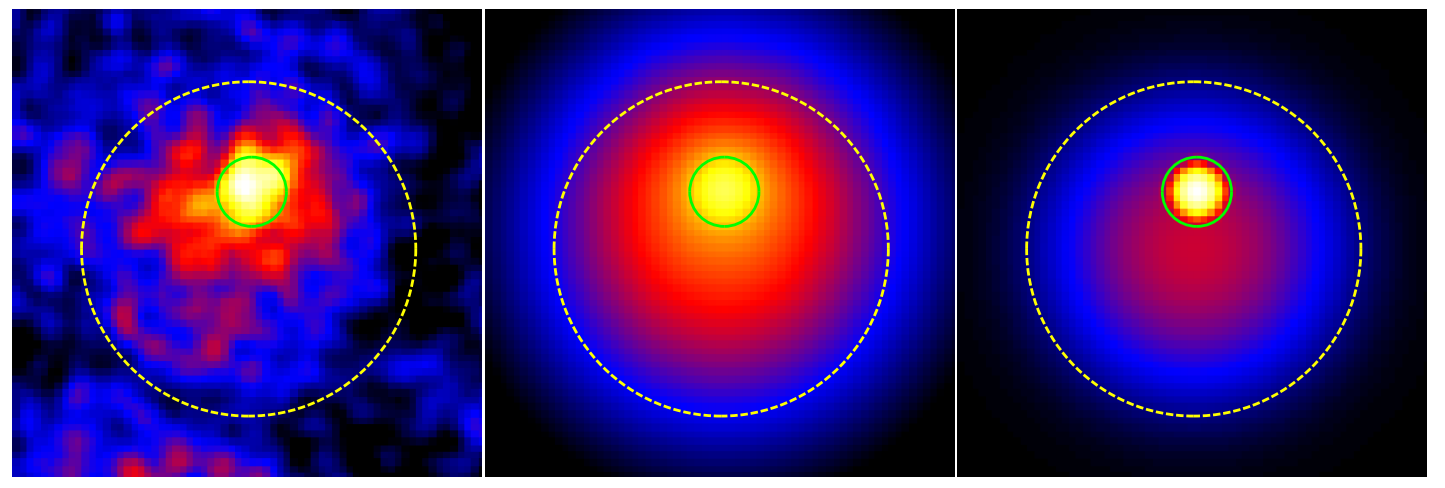

Figure 1: An example of 2D image fitting procedure of point-like Arches cluster's core thermal emission (2D Gaussian model $G 1,15^{\prime}$ radius green circle) and the extended non-thermal emission of the surrounding molecular cloud (2D Gaussian model G2, yellow dashed 72".4 FWHM radius circle). Left: NuSTAR image of the Arches cluster complex in $5.8-7.2 \mathrm{keV}$ band, compatible with fluorescent iron line emission of the cloud (G2) at $6.4 \mathrm{keV}$ and emission line of ionized iron at energy $6.7 \mathrm{keV}$ of the cluster (G1). Middle: Best-fit spatial model of the Arches cluster complex in $5.8-7.2 \mathrm{keV}$ band with two gaussians $G 1$ and $G 2$ convolved with the NuSTAR PSF. Right: The same as middle plot, but not convolved with the PSF.

By running the fitting procedure in each of the 15 energy bands, we estimated the best-fitting parameters of the spatial models $G 1$ and $G 2$. We then calculated the flux, i.e. amount of collected photons with each model divided by the exposure time. The corresponding errors were estimated from multi-variate normal distribution, based on sampling the set of thawed parameters and calculating the model flux (normal_sample function in SHERPA). The estimated model fluxes and 
uncertainties were combined into PHA spectra files of each spatial model $G 1$ and $G 2$ shown in Fig. 2. The corresponding Redistribution Matrix File (RMF), which maps from energy space into PHA space, was simply adopted from the standard spectral analysis of the 'calibration' source PMN J0641-0320 (see below) with nuproducts and rebinned with rbnrmf tool of HEASOFT 6.19 package.

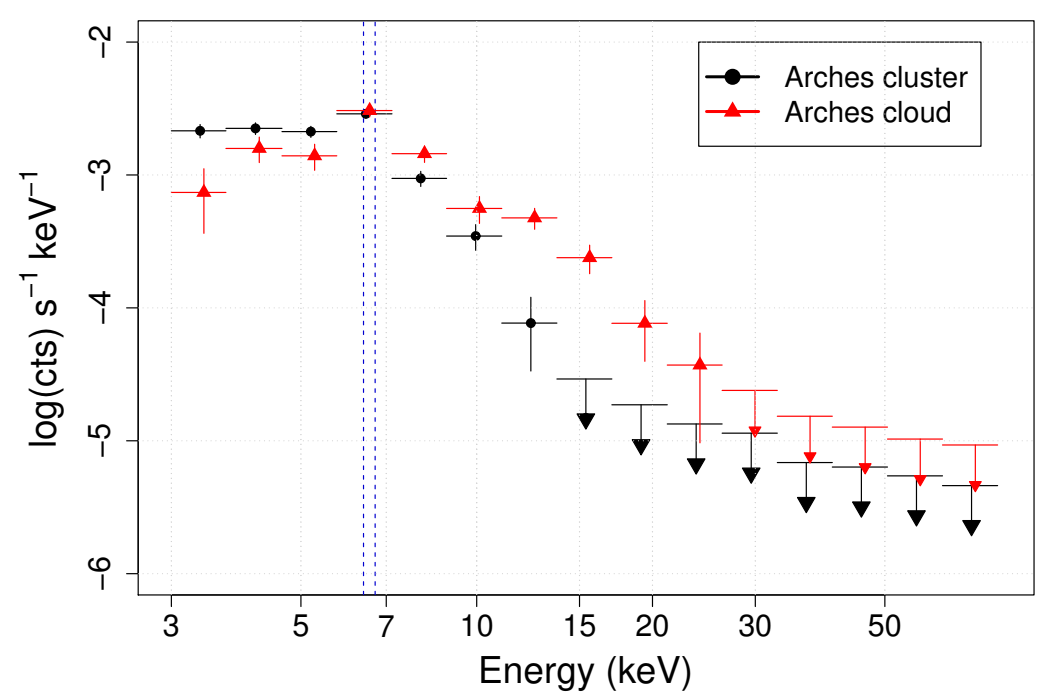

Figure 2: Spatially resolved NUSTAR spectra of the Arches cluster core (black) and cloud (red) X-ray emission. The position of Fe $\mathrm{K} \alpha 6.4$ and $6.7 \mathrm{keV}$ are marked by vertical dashed lines, as in the NuSTAR standard spectra these two lines cannot be separated by our technique. The upper limits are $1 \sigma$ errors.

As seen from Fig. 2, spatially confused emission of the Arches cluster complex was effectively decoupled with 2D image fitting procedure into the soft thermal emission of the cluster and hard non-thermal emission of the molecular cloud. Since the applied method uses the full collective power of the PSF, we could detect hard X-ray emission up to $20-30 \mathrm{keV}$. X-ray emission of the cluster contains an excess in the $5.8-7.2 \mathrm{keV}$ range, compatible with $\sim 6.7 \mathrm{keV}$ line and rapidly drops above $\sim 10 \mathrm{keV}$ as expected for thermal emission with $k T \approx 2 \mathrm{keV}$. Non-thermal emission of the extended cloud component apparently includes excess around $6.4 \mathrm{keV}$ and dominates above $10 \mathrm{keV}$.

To use spatially decoupled $G 1$ and $G 2$ spectra in XSPEC spectral modeling package we calibrated effective area (ARF) utilizing the NUSTAR data of a bright source with known spectrum. To this end we used 20 ks observation (ObsID: 80001003002) of MeV Blazar PMN J0641-0320 with very hard power-law spectrum of $\Gamma \approx 1$ detectable up to $\sim 80 \mathrm{keV}$ [17]. We have done standard spectral extraction (Sect. 2) of PMN J0641-0320 within 70" radius circle, and estimated source flux phot $\mathrm{s}^{-1} \mathrm{~cm}^{-2}$ in each of the 15 energy bands. We then repeated 2D image spectral extraction procedure (Sect. 3) for PMN J0641-0320 as a point-like source (one 2D Gaussian with 4" FWHM size) and estimated model flux phot $\mathrm{s}^{-1}$ in each of the 15 energy bands. Comparing the model fluxes obtained by two methods, we extracted the effective area in $\mathrm{cm}^{-2}$ for each band and combined them into ARF file. We should note that here we assume that the ARF calibrated for a point-like source is suitable for the extended emission of the Arches cloud. Given the limited 
statistics of the NUSTAR Arches cluster observations, the deviations are within the uncertainties.

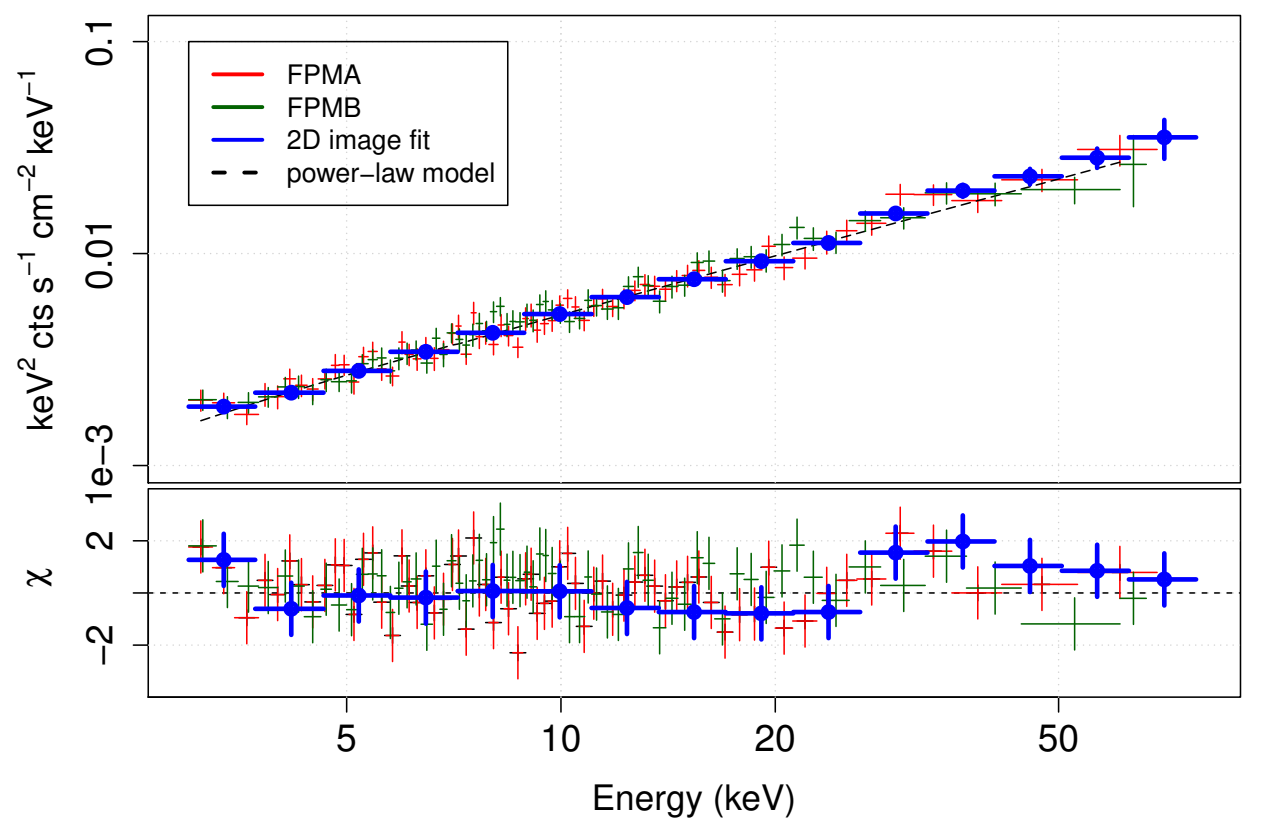

Figure 3: NUSTAR spectrum of MEV blazar PMN J0641-0320 extracted from $R=70^{\prime}$ circular region with standard procedures (red and green), and spectrum obtained through 2D image analysis (blue) described in this paper.

Fig. 3 shows X-ray spectra of MeV Blazar PMN J0641-0320 obtained with standard method (Sect. 2) for two NuSTAR modules FPMA and FPMB, and combined FPMA+FPMB spectrum extracted with 2D image algorithm (Sect. 3). It is seen that spectra are consistent with each other, demonstrating reliability of the 2D image fitting approach.

\section{Results}

The final spatially decoupled spectra of the Arches stellar cluster and extended molecular cloud emission is shown in Fig. 4. We fitted the stellar cluster emission spectrum with one APEC model subjects to a line-of-sight photoelectric absorption fixed at $N_{\mathrm{H}}=9.5 \times 10^{22} \mathrm{~cm}^{-2}$. This simple model provides an acceptable fit to the data with $\chi_{\mathrm{r}}^{2} /$ d.o.f. $=0.66 / 13$. We estimated two parameters from the fit: the temperature of the plasma $k T=2.44 \pm 0.40 \mathrm{keV}$ and the unabsorbed $3-8 \mathrm{keV}$ flux $(8.70 \pm 0.70) \times 10^{-13} \mathrm{erg} \mathrm{s}^{-1} \mathrm{~cm}^{-2}$.

The emission of the Arches cluster extended emission was approximated with power-law model and a Gaussian line with position and width fixed at $6.4 \mathrm{keV}$ and $0.1 \mathrm{keV}$, respectively. The model gives acceptable fit statistics $\chi_{\mathrm{r}}^{2} /$ d.o.f. $=1.02 / 12$ and allows for a constraint on the power-law slope $\Gamma=2.06 \pm 0.26$ and the unabsorbed $3-20 \mathrm{keV}$ flux $F_{3-20}=(9.33 \pm 1.34) \times$ $10^{-13} \mathrm{erg} \mathrm{s}^{-1} \mathrm{~cm}^{-2}$. The total flux of the $6.4 \mathrm{keV}$ Gaussian line was estimated to be $(1.38 \pm$ $0.50) \times 10^{-5}$ photons $\mathrm{cm}^{-2} \mathrm{~s}^{-1}$. 

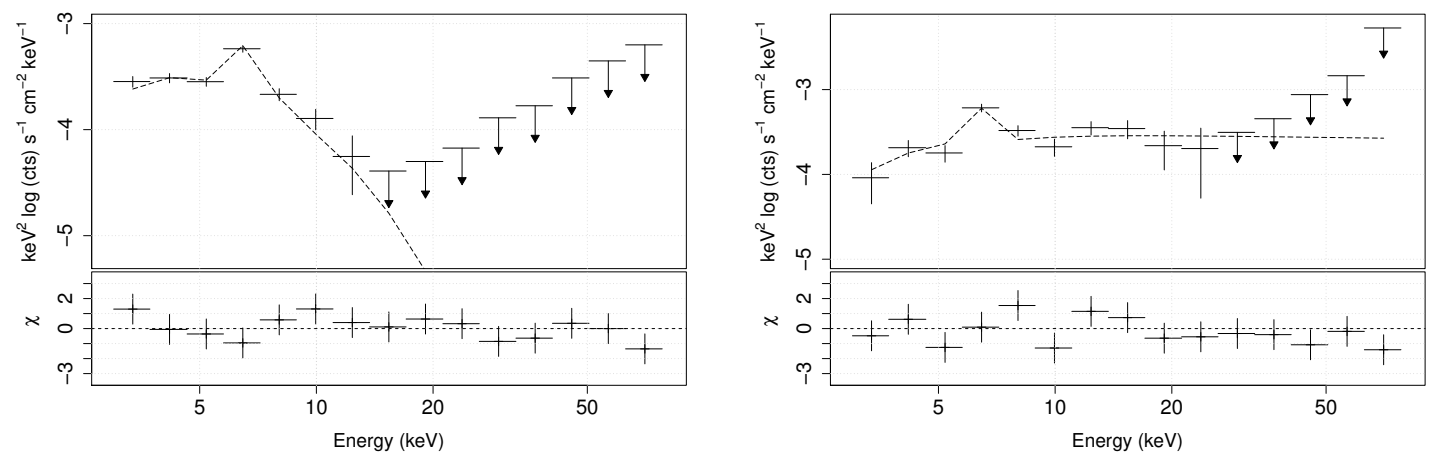

Figure 4: Spatially decoupled NUSTAR spectrum of the Arches cluster complex obtained with 2D image fitting procedure. Left: thermal spectrum of the Arches stellar cluster (APEC, $k T \sim 2 \mathrm{keV})$. Right: nonthermal X-ray spectrum of the Arches molecular cloud extended emission, approximated with power-law model and iron $6.4 \mathrm{keV}$ line represented with Gaussian.

\section{Summary}

In this work we presented details of the 2D image analysis approach used to decouple spatially confused emission components in the Arches cluster complex [12], namely, thermal emission of the stellar cluster and surrounding extended non-thermal emission of the molecular cloud. We demonstrated the capability of the method to decouple spatial emission components and validated its reliability in comparison with standard approach.

\section{Acknowledgments}

RK acknowledges support from the Russian Basic Research Foundation (grant 16-02-00294), the Academy of Finland (grant 300005) and hospitality of the Tuorla Observatory.

\section{References}

[1] Chlebowski, T., \& Garmany, C. D. 1991, ApJ, 368, 241

[2] Yusef-Zadeh, F., et al. 2002, ApJ, 570, 665

[3] Wang, Q. D., Dong, H., \& Lang, C. 2006, MNRAS, 371, 38

[4] Capelli, R., et al. 2011a, A\&A, 525, L2

[5] Figer, D. F., et al. 1999, ApJ, 525, 750

[6] Tsujimoto, M., Hyodo, Y., \& Koyama, K. 2007, PASJ, 59, 229

[7] Capelli, R., et al. 2011b, A\&A, 530, A38

[8] Tatischeff, V., Decourchelle, A., \& Maurin, G. 2012, A\&A, 546, A88
[9] Krivonos R. A., et al., 2014, ApJ, 781, 107

[10] Clavel M., et al. 2014, MNRAS, 443, L129

[11] Harrison, F. A., et al. 2013, ApJ, 770, 103

[12] Krivonos, R., et al. 2017, arXiv:1612.03320

[13] Krivonos, R., \& Sazonov, S. 2016, MNRAS, 463,756

[14] Arnaud, K. A. 1996, Astronomical Data Analysis Software and Systems V, 101, 17

[15] Freeman, P., Doe, S., \& Siemiginowska, A. 2001, Proc. SPIE, 4477, 76

[16] Fruscione, A., et al. 2006, Proc. SPIE, 6270,

[17] Ajello, M., et al. 2016, ApJ, 826, 76 